\title{
Limit-cycle oscillatory coexpression of cross-inhibitory transcription factors: a model mechanism for lineage promiscuity
}

\author{
Pavol Bokes ${ }^{1} \quad$ John R. King ${ }^{2}$ \\ ${ }^{1}$ Department of Applied Mathematics and Statistics, Comenius \\ University, Bratislava 842 48, Slovakia \\ ${ }^{2}$ School of Mathematical Sciences and SBRC Nottingham, \\ University of Nottingham, Nottingham NG7 2RD, United \\ Kingdom
}

\begin{abstract}
Lineage switches are genetic regulatory motifs that govern and maintain the commitment of a developing cell to a particular cell fate. A canonical example of a lineage switch is the pair of transcription factors PU.1 and GATA-1, of which the former is affiliated with the myeloid and the latter with the erythroid lineage within the hematopoietic system. On a molecular level, PU.1 and GATA-1 positively regulate themselves and antagonise each other via direct protein-protein interactions. Here we use mathematical modelling to identify a novel type of dynamic behaviour that can be supported by such a regulatory architecture. Guided by the specifics of the PU.1-GATA-1 interaction, we formulate, using the law of mass action, a system of differential equations for the key molecular concentrations. After a series of systematic approximations, the system is reduced to a simpler one, which is tractable to phase-plane and linearisation methods. The reduced system formally resembles, and generalises, a well-known model for competitive species from mathematical ecology. However, in addition to the qualitative regimes exhibited by a pair of competitive species (exclusivity, bistable exclusivity, stable-node coexpression) it also allows for oscillatory limit-cycle coexpression. A key outcome of the model is that, in the context of cell-fate choice, such oscillations could be harnessed by a differentiating cell to prime alternately for opposite outcomes; a bifurcation-theory approach is adopted to characterise this possibility.
\end{abstract}

\section{Introduction}

\subsection{Lineage selection and multilineage priming}

The differentiation of hematopoietic stem cells into mature blood cells consists of a series of branching decisions, which successively restrict the availability of certain cell fates, and enforce others (Akashi et al., 2000; Nimmo et al., 2015). Any such decision is thought to be regulated by a lineage switch, a genetic 
regulatory motif which typically consists of two mutually inhibiting transcription factors (Swiers et al., 2006). Which of these is turned on determines which branch of the hematopoietic decision tree is selected; cross-inhibition guarantees the exclusivity of commitment (Cantor and Orkin, 2001).

The transcription factors PU.1 and GATA-1 are key hematopoietic regulators that are associated with myeloid and erythroid lineages, respectively (Shivdasani and Orkin, 1996). Either factor is able to maintain its expression via positive transcriptional feedback (Chen et al., 1995; McDevitt et al., 1997). However, PU.1 interferes with GATA-1's autoregulation by interacting with the latter's DNA binding region (Zhang et al., 2000). Conversely, GATA-1 inactivates PU.1 by binding to a region of PU.1 that would otherwise be available to its critical co-activator c-Jun (Zhang et al., 1999). Provided that these antagonistic interactions are sufficiently strong, the ability of either factor to sustain itself via a positive feedback loop is contingent on the absence of its antagonist, resulting in switch-like behaviour (Graf, 2002). Other examples of lineage switches are Gfi-1 v. Egr within the myeloid compartment (Laslo et al., 2006) and EKLF v. Fli-1 within the erythroid compartment (Krumsiek et al., 2011). On a different branch of the decision tree, T-bet v. GATA-3 dictates commitment in T cells (Antebi et al., 2013).

The availability of multiple alternative long-time outcomes (in the form of stable steady states) is a crucial feature of mathematical representations for lineage selection. Bistability in a lineage switch means that the choice of lineage depends on which factor holds an advantage initially (e.g. Waters et al., 2017). A commitment decision can be reversed by a transient forced expression of the transcription factor associated with the alternative genetic programme (Kulessa et al., 1995; Nerlov and Graf, 1998). Bistability (or more general multistability) can explain such reversals of commitment in the form of an escape from the basin of attraction of the chosen stable state driven by an application of external forcing.

Prior to their commitment to a particular lineage, bipotent progenitor cells coexpress genes of both available lineage programmes, in a phenomenon bearing the names of multilineage priming or lineage promiscuity (Nimmo et al., 2015; Hu et al., 1997). It has been suggested that the coexpression could be either simultaneous, in which case the propensity for one or other programme is constant over time, or fluctuating, meaning that the cell alternatingly primes for either lineage (Hu et al., 1997). Simultaneous lineage promiscuity can be modelled by lineage switches in weakly cross-inhibiting regimes possessing a stable coexpression steady state (Laslo et al., 2006). Lineage promiscuity has also been modelled by other approaches, including: a coexpression state of a switch supporting a tristable regime (Huang et al., 2007; Foster et al., 2009); by a burst-like stochastic constitutive expression of lineage-affiliated transcription factors (Teles et al., 2013); by metastable attractors in a stochastic model emerging as a result of including explicit mRNA dynamics (Strasser et al., 2012); and through using the bistable regime in excessive noise conditions (Bokes et al., 2013); the need for further modelling methodologies has been accentuated in recent experimental studies challenging prevailing approaches (Hoppe et al., 2016; Buggenthin et al., 2017; Velten et al., 2017). 


\section{$1.2 \quad$ Modelling assumptions}

Differing interpretations and emphases on the specifics of the PU.1 and GATA-1 example have led to a variety of alternative mathematical models, a number of which are reviewed in (Duff et al., 2012); see also (Tian and Smith-Miles, 2014; Alsaedi et al., 2014) for models incorporating the GATA-2 factor.

While most models for PU.1 and GATA-1 are based on the Shea-Ackers formalism (Shea and Ackers, 1985; Bintu et al., 2005) and consider the effects of the interaction at the genes' promoters only, we proposed an alternative approach (Bokes et al., 2009) according to which any molecular pair can interact: only a fraction of the total protein number is free (transcriptionally active), while the rest are engaged in a disabling protein-protein complex. Yet the proteinprotein interaction is not permanent; on the contrary, the free and bound groups are continuously exchanging constituents, old interactions being ceaselessly torn apart and replaced by new ones. Similar approaches have been used elsewhere to describe the interaction of a protein with DNA decoy binding sites (Lee and Maheshri, 2012; Burger et al., 2010; Bokes and Singh, 2015) and protein dimerisation (Erban et al., 2006).

Since GATA-1 deactivates PU.1 but does not disrupt the latter's ability to bind to the DNA (Zhang et al., 1999), there is a possibility that a GATA-1 - PU.1 complex may effectively repress the $p u .1$ gene by displacing free PU.1 molecules from the promoter (Chickarmane et al., 2009). Aiming to keep our model as simple as possible, in (Bokes et al., 2009) we ignored (and will continue to do so here) the structural asymmetry of the PU.1 and GATA-1 interaction, using PU.1's effect on GATA-1 as a template for a more generic, structurally symmetric, model. In part for this reason we shall refer to our model's antagonists as $\mathrm{X}_{1}$ and $\mathrm{X}_{2}$ rather than restricting ourselves to specific transcription factor names.

As is customary in models of this kind, we assume that proteins are degraded with a rate that increases linearly with their concentration (Alon, 2007; Tyson et al., 2003). Complexed proteins must also be degraded, lest the proteinprotein interaction should serve merely as a reservoir of decay-proof molecules, unable to maintain the necessary competitive pressure. Previously in (Bokes et al., 2009), we made the protein-protein complexes degrade as a whole, implying that there should be a single mechanism which removes both constituent proteins simultaneously. Here we take a different stance, assuming instead that there are two separate degradation mechanisms, each degrading one of the factors while freeing its partner. This new approach, as well as being biologically sound, will have an additional advantage of removing a mathematically superfluous constraint in the parameter space of our model. It is within this extension of the parameter space that a new type of qualitative behaviour will be found.

\subsection{Paper's Outline}

The model is derived and systematically simplified in Section 2. First, we express our key modelling assumptions in the language of the law of mass action, which yields a system of three differential equations, two for the transcription factor antagonists in free form and one for their complex. The system is nondimensionalised, which helps identify the crucial dimensionless parameter groupings, several of which can reasonably be assumed to be small. Neglecting the small 
terms in the usual fashion, we end up with a simpler, tractable, system of two differential equations, the equation for the complex being replaced in one of the simplifications by an algebraic relation.

The reduced two-dimensional system formally generalises (in a sense made explicit in Section 2) a classical ecological model for the population dynamics of two competing species (Murray, 2003). Phase-plane and linearisation analyses of the competitive species model help identify three distinct regimes of qualitative behaviour (Murray, 2003):

Exclusivity The stronger competitor (transcription factor/ecological species) inevitably defeats the weaker and ends up reigning unopposed.

Bistability Both competitors are strong: initial advantage determines which wins and which disappears.

Coexistence/coexpression Weak competition makes the simultaneous presence of the competitors possible.

Extending in Section 3 the qualitative analysis of competitive species to our genetic switch model opens up an additional possibility of

Oscillatory coexpression The transcription factors are alternately favoured due to weak but asymmetric competition.

Oscillations in the concentrations of transcription factors have previously been shown to result from production delays (Mitra et al., 2014; Cao et al., 2016) and to occur due to diseases (Ruggieri et al., 2012). We show that, in our model, the regime of oscillatory coexpression is available only if certain criteria of parametric asymmetry are met. Identifying these criteria in Section 3 helps us understand intuitively how the oscillations are first triggered and then sustained.

The model can change its regime from oscillatory coexpression to bistable due to specific changes to parameter values, which are introduced in Section 4. We also show that the phase of the oscillation at the point of parametric change can determine which of the two stable steady states that are available in the bistable regime is selected. In Section 5, we investigate the bifurcation structure of the model. The results of the paper are summarised and placed within a wider context in Section 6.

\section{Modelling framework}

We consider a system of three ordinary differential equations for the concentrations $x_{1}$ of free factor $\mathrm{X}_{1}$ (e.g. PU.1), $x_{2}$ of free factor $\mathrm{X}_{2}$ (e.g. GATA-1), and $y$ of their complex Y,

$$
\begin{aligned}
\frac{\mathrm{d} x_{1}}{\mathrm{~d} t} & =\frac{p_{1} x_{1}}{K_{1}+x_{1}}-\alpha_{1} x_{1}+\beta_{2} y-k_{\text {on }} x_{1} x_{2}+k_{\text {off }} y, \\
\frac{\mathrm{d} x_{2}}{\mathrm{~d} t} & =\frac{p_{2} x_{2}}{K_{2}+x_{2}}-\alpha_{2} x_{2}+\beta_{1} y-k_{\text {on }} x_{1} x_{2}+k_{\text {off }} y, \\
\frac{\mathrm{d} y}{\mathrm{~d} t} & =k_{\mathrm{on}} x_{1} x_{2}-\left(k_{\mathrm{off}}+\beta_{1}+\beta_{2}\right) y .
\end{aligned}
$$


The first two terms on the right-hand sides of (1)-(2) are the production and decay rates of free transcription factors. The production rate exhibits a MichaelisMenten type dependence on the protein concentration, which is indicative of noncooperative positive autoregulation (Keener and Sneyd, 2008); the decay rates are proportional to the protein concentrations. The remaining mass-action expressions in (1)-(3) represent the reactions

$$
\mathrm{X}_{1}+\mathrm{X}_{2} \underset{k_{\text {off }}}{\stackrel{k_{\text {on }}}{\rightleftharpoons}} \mathrm{Y}, \quad \mathrm{Y} \stackrel{\beta_{1}}{\longrightarrow} \mathrm{X}_{2}, \quad \mathrm{Y} \stackrel{\beta_{2}}{\longrightarrow} \mathrm{X}_{1},
$$

the first of which is the reversible pair of complexification and dissociation, and the other two (irreversible) reactions represent the decay of protein in complexed form; note that if a complexed protein is degraded, its partner is freed. Hence, complexification can affect the constituent proteins twofold: first, it interferes with their ability to bind to the promoter to catalyse the expression of their gene; second, it changes their rate of decay from $\alpha_{1}\left(\alpha_{2}\right)$ to $\beta_{1}\left(\beta_{2}\right)$.

Adding the equation for the complex (3) to those for the free protein (1)-(2), we obtain

$$
\frac{\mathrm{d}\left(x_{i}+y\right)}{\mathrm{d} t}=\frac{\alpha_{i} x_{i}\left(\frac{p_{i}}{\alpha_{i}}-K_{i}-x_{i}\right)}{K_{i}+x_{i}}-\beta_{i} y, \quad i=1,2
$$

for total concentrations of protein — both free and complexed. Also, we expressed in (5) the difference of production and decay rates in the form of a single rational function. Equations (5), if supplemented by equation (3) for the protein complex, can be used as an equivalent, and in a number of aspects more convenient, formulation of system (1)-(3).

The model given by (5) and (3) is nondimensionalised and is simplified in Appendix A. The simplification consists of two steps: (i) we use a quasi-steadystate approximation for protein complexification and dissociation; (ii) we assume that the autoregulation of either factor operates far below its saturation limit. We obtain thereby a planar system for the normalised protein concentrations $u_{1}$ and $u_{2}$ as functions of the normalised time $\tau$ (A1), which reads

$$
\begin{aligned}
& \frac{\mathrm{d}\left(u_{1}+b_{12} u_{1} u_{2}\right)}{\mathrm{d} \tau}=u_{1}\left(1-u_{1}-a_{12} u_{2}\right), \\
& \frac{\mathrm{d}\left(u_{2}+b_{21} u_{1} u_{2}\right)}{\mathrm{d} \tau}=\rho u_{2}\left(1-u_{2}-a_{21} u_{1}\right) .
\end{aligned}
$$

The system (6)-(7) depends on five dimensionless parameters: $a_{12}, a_{21}, b_{12}, b_{21}$, and $\rho$; their relation to the dimensional parameters is given by (A5) and (A6), but their meaning is explained below.

In a special case $b_{12}=b_{21}=0$, the system (6)-(7) reduces, in its mathematical form, to a well-studied ecological model for two competitive species (Murray, 2003). The phase-plane analysis of the competitive species model implies that, in particular, any nondegenerate steady states must be saddles or nodes, but not spirals, and that no limit cycles may exist (Hirsch, 1982). In the next sections, we will show that such restrictions are no longer in place if $b_{12}$ and $b_{21}$ are allowed to be nonzero.

In an analogy with competitive species, the parameters $a_{12}$ and $a_{21}$ measure the competitive impact of the second transcription factor on the first and vice 
versa, respectively. The parameters $b_{12}$ and $b_{21}$ measure the ability of the second factor to bind the first and vice versa, respectively. Indeed, should we perturb the system from the state of exclusive expression of the second factor by adding a small amount of the first factor, then $b_{12}$ gives the ratio of complexed and free molecules of the added factor; a symmetric statement can of course be made for $b_{21}$. The aforementioned special choice of $b_{12}=b_{21}=0$ is that of competition by annihilation: while negligible amounts of either factor are bound in a complex at any time, the ephemeral interaction strongly catalyses their degradation. The parameter $\rho$ compares the growth rates of the two antagonists.

Chain-rule differentiating the left-hand sides of (6)-(7) and solving in $\mathrm{d} u_{1} / \mathrm{d} \tau$ and $\mathrm{d} u_{2} / \mathrm{d} \tau$ yields

$$
\begin{aligned}
\frac{\mathrm{d} u_{1}}{\mathrm{~d} \tau} & =\frac{\left(1+b_{21} u_{1}\right) f_{1}\left(u_{1}, u_{2}\right)-b_{12} u_{1} f_{2}\left(u_{1}, u_{2}\right)}{1+b_{12} u_{2}+b_{21} u_{1}}, \\
\frac{\mathrm{d} u_{2}}{\mathrm{~d} \tau} & =\frac{-b_{21} u_{2} f_{1}\left(u_{1}, u_{2}\right)+\left(1+b_{12} u_{2}\right) f_{2}\left(u_{1}, u_{2}\right)}{1+b_{12} u_{2}+b_{21} u_{1}},
\end{aligned}
$$

in which

$$
f_{1}\left(u_{1}, u_{2}\right)=u_{1}\left(1-u_{1}-a_{12} u_{2}\right), \quad f_{2}\left(u_{1}, u_{2}\right)=\rho u_{2}\left(1-u_{2}-a_{21} u_{1}\right)
$$

are the right-hand sides of (6)-(7). The explicit form (8)-(10) is useful for numerical simulations, whereas the implicit form (6)-(7) is preferable for analysis.

\section{Phase-plane analysis}

Steady-state solutions are obtained by equating the right-hand sides of (6) and $(7)$ to zero. The zero steady state $(0,0)^{\top}$ is always a stable node (see Appendix B for linearisation analysis around this and other steady states). The steady state $(1,0)^{\top}$ of $\mathrm{X}_{1}$-exclusivity is a stable node if $a_{21}>1$ and a saddle if $a_{21}<1$. Conversely, $(0,1)^{\top}$ is a stable node if $a_{12}>1$ and a saddle if $a_{12}<1$. The exclusive expression states, should they be saddles, are stable with respect to perturbations in the concentration of the factor that is being exclusively expressed, but unstable with respect to perturbations that add but a minute amount of the antagonist.

If $a_{21}>1$ and $a_{12}<1$, then $(1,0)^{\top}$ is a single global attractor (Figure 1, centre left panel); conversely, if $a_{21}<1$ and $a_{12}>1$, it is $(0,1)^{\top}$ that attracts all positive initial conditions. If $a_{12}>1$ and $a_{21}>1$, then the exclusive expression steady states are both stable nodes. There is an additional, co-expression, steady state with coordinates

$$
\bar{u}_{1}=\frac{1-a_{12}}{1-a_{12} a_{21}}, \quad \bar{u}_{2}=\frac{1-a_{21}}{1-a_{12} a_{21}},
$$

which satisfy $\bar{u}_{1}+\bar{u}_{2}<1$. Linearisation analysis shows this to be a saddle. The stable manifold (here a curve) of the saddle is the separatrix, delineating the basins of attractions to the two stable nodes of exclusive expression (Figure 1, centre right panel).

If $a_{12}<1$ and $a_{21}<1$, then the exclusive expression steady states are both saddles. The co-expression steady state (11) is available again, but satisfies 

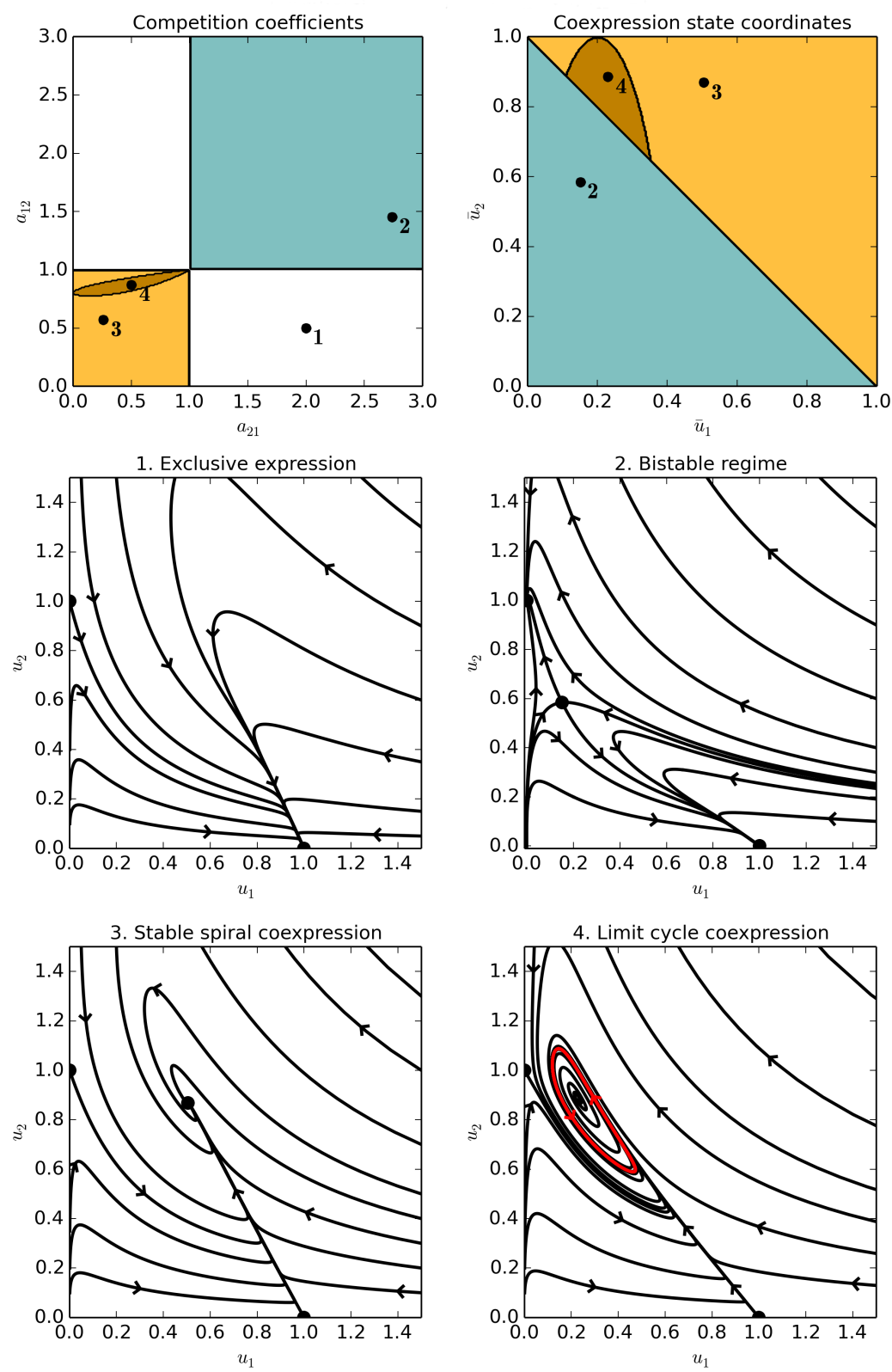

Figure 1: A selection of phase portraits of (6)-(7). The binding abilities are $b_{21}=5, b_{12}=1$; the growth rate ratio is $\rho=0.2$. Four different combinations of the competition coefficients $a_{12}$ and $a_{21}$ are selected: (1) $a_{21}=2$, $a_{12}=0.5 ;(2) a_{21}=2.74, a_{12}=1.45 ;(3) a_{21}=0.26, a_{12}=0.57 ;$ (4) $a_{21}=0.5$, $a_{12}=0.87$. Shadings in the top panels indicate individual parametric regimes: bistable (blue), stable-steady-state coexpression (yellow), oscillatory coexpression (brown). Note that there is a one-to-one correspondence between the competition strengths $a_{12}$ and $a_{21}$ and the coexpression steady state coordinates $\bar{u}_{1}$ and $\bar{u}_{2}$. 
$\bar{u}_{1}+\bar{u}_{2}>1$. Linearisation shows that it can be a node or a spiral, and that it is unstable if

$$
\rho \bar{u}_{2}\left(b_{12}\left(1-2 \bar{u}_{2}\right)-1\right)+\bar{u}_{1}\left(b_{21}\left(1-2 \bar{u}_{1}\right)-1\right)>0 .
$$

The Poincare-Bendixson theorem then guarantees that there exists a limit cycle, which substitutes as a global attractor for the unstable steady states (Figure 1, bottom right panel). For fixed values of $b_{12}, b_{21}$ and $\rho$, condition (12) represents an ellipse in the $\left(\bar{u}_{1}, \bar{u}_{2}\right)$-plane (Figure 1, upper right panel, brown region), which corresponds via (11) to a specific region in the $\left(a_{21}, a_{12}\right)$-plane (Figure 1 , upper left panel, brown region). If the left-hand side of (12) is, on the contrary, negative, then the coexpression steady state is stable and attracts all positive initial conditions (Figure 1, bottom left panel).

Limit-cycle oscillations can be sustained by our model only in certain parametric regions of the coexpression regime, which are defined by specific requirements on asymmetry. We call the competitors equally strong if $a_{12}=a_{21}$. If $a_{12}>a_{21}$, then the second factor is the stronger and the first is the weaker competitor; if $a_{21}>a_{12}$ it is the other way round. Since $\bar{u}_{1} / \bar{u}_{2}=\left(1-a_{12}\right) /\left(1-a_{21}\right)$, the weaker competitor is expressed at a lower level - relative to the maximal self-sustainable expression - than the stronger competitor. In order to sustain oscillatory coexpression, the weaker competitor needs to be expressed at less than its half-maximal level to make either $1-2 \bar{u}_{1}$ or $1-2 \bar{u}_{2}$ positive in (12); since $\bar{u}_{1}+\bar{u}_{2}>1$ holds in the coexpression scenario, the stronger one must be expressed at more than its half-maximal level. In particular, equally strong competitors cannot sustain oscillations.

Without loss of generality, we assume that the first factor is the weaker competitor. In the coexpression regime we have $1-2 \bar{u}_{2}<2 \bar{u}_{1}-1$; condition (12) implies that $\left(1-2 \bar{u}_{1}\right)\left(b_{21} \bar{u}_{1}-\rho b_{12} \bar{u}_{2}\right)-\rho \bar{u}_{2}-\bar{u}_{1}>0$, and therefore $b_{21} \bar{u}_{1}>$ $\rho b_{12} \bar{u}_{2}$, i.e. $\rho b_{12} / b_{21}<\bar{u}_{1} / \bar{u}_{2}<1$. The latter means that the weaker competitor should have the stronger growth rate and/or the greater binding ability. On the other hand, we have $1<a_{12} / a_{21}=\beta_{1} \rho b_{12} / \beta_{2} b_{21}$ (compare (A5) and (A6)), which together with $\rho b_{12} / b_{21}<1$ implies that $\beta_{1}>\beta_{2}$ : hence, the weaker competitor must be degraded faster in complexed form.

In Figure 2, time traces are shown of the competing transcription factors' concentrations for parameter values which conform to the regime of oscillatory coexpression (Figure 1, bottom right). In the top panel of Figure 2, the normalised free protein concentrations $u_{1}$ and $u_{2}$ are shown in solid blue and orange, while the total protein concentrations $u_{1 \mathrm{~T}}=u_{1}+b_{12} u_{1} u_{2}$ and $u_{2 \mathrm{~T}}=u_{2}+b_{21} u_{1} u_{2}$ are represented by dashed lines.

The transcription factors' normalised concentrations are measured relatively to their maximal possible self-sustainable expression levels, which can differ between the two factors. In the bottom panel of Figure 2, we compensate for the difference in scales by multiplying the normalised concentrations by their binding abilities $b_{21}$ and $b_{12}(\mathrm{~A} 6)$. The compensation is equivalent to measuring both protein concentrations in common units of their interaction's dissociation constant $K_{\mathrm{d}}=k_{\mathrm{off}} / k_{\mathrm{on}}$. The usage of a common unit of concentration emphasises that the weaker competitor is the more abundant one (Figure 2, bottom).

Crucially, the weaker competitor's free and total concentrations oscillate in sync, but there is a distinct phase shift between the free and total concentrations of the stronger competitor (Figure 2). In the specific example shown in Figure 2, 

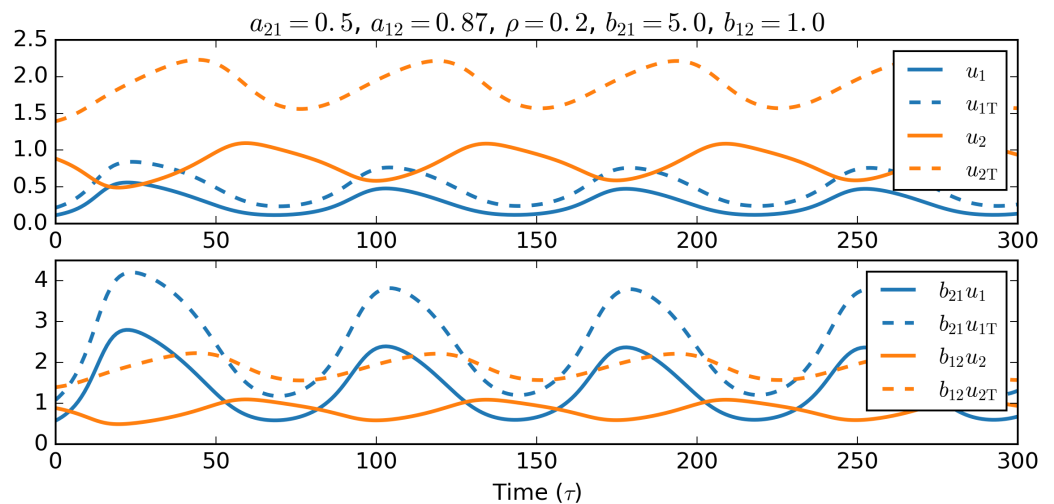

Figure 2: Oscillatory coexpression of competitive transcription factors: time traces of free (full line) and total (dashed line) concentrations for both the weaker (blue colour) and the stronger (orange colour) competitors.

the weaker competitor starts below its coexpression steady-state level, and the (unbound) stronger competitor is initially above its steady-state level. The undervalued weaker competitor grows and, being an efficient binder, captures the stronger competitor into a complex; thus, the amount of free molecules $u_{2}$ decreases although at the same time the total molecule level $u_{2 \mathrm{~T}}$ increases. Having captured much of its opponent, the weaker competitor peaks at a high value. The peak is followed by a corrective phase, during which the weaker competitor decays and the stronger competitor grows. The growth is driven, in the last stages, by the release of the captured molecules: the total molecular amount $u_{2 \mathrm{~T}}$ decreases while $u_{2}$ increases further, eventually peaking at a high value. From this peak, another round of oscillation commences. Thus, the oscillatory dynamics are driven by the capture and release of the stronger competitor by the weaker competitor.

\section{Lineage choice driven by phase}

Here we assume that for $0<\tau<\tau_{\mathrm{c}}$ the transcription factor concentrations satisfy the system (6)-(7) with competition coefficients in the coexpression regime $a_{12}<1$ and $a_{21}<1$. Additionally, we require that $a_{12} \neq a_{21}$ and that the remaining dimensionless parameters $b_{21}, b_{12}$ and $\rho$ be chosen so as to render the coexpression oscillatory. At $\tau=\tau_{c}$, the cell receives a specific signal for lineage commitment.

In response to the signal, the competition strength and the binding ability of either factor are assumed to increase proportionally (here and below we use tilded symbols for values after the change)

$$
\tilde{a}_{21}=\lambda_{1} a_{21}, \quad \tilde{b}_{21}=\lambda_{1} b_{21}, \quad \tilde{a}_{12}=\lambda_{2} a_{12}, \quad \tilde{b}_{12}=\lambda_{2} b_{12} .
$$

We require $\tilde{a}_{12}>1$ and $\tilde{a}_{21}>1$ (i.e. $\lambda_{2}>1 / a_{12}$ and $\lambda_{1}>1 / a_{21}$ ) in order that the changed parameters belong to the bistable regime. 
For $\tau>\tau_{\mathrm{c}}$, the transcription factor concentrations satisfy the same system as before but for the change in parameters, i.e.

$$
\begin{aligned}
& \frac{\mathrm{d}\left(\tilde{u}_{1}+\tilde{b}_{12} \tilde{u}_{1} \tilde{u}_{2}\right)}{\mathrm{d} \tau}=\tilde{u}_{1}\left(1-\tilde{u}_{1}-\tilde{a}_{12} \tilde{u}_{2}\right), \\
& \frac{\mathrm{d}\left(\tilde{u}_{2}+\tilde{b}_{21} \tilde{u}_{1} \tilde{u}_{2}\right)}{\mathrm{d} \tau}=\rho \tilde{u}_{2}\left(1-\tilde{u}_{2}-\tilde{a}_{21} \tilde{u}_{1}\right) .
\end{aligned}
$$

The concentrations $\tilde{u}_{1}$ and $\tilde{u}_{2}$ in (14)-(15) are measured in units of their maximal self-sustainable levels; we assume that these also increase with the proportionality factors $\lambda_{1}$ and $\lambda_{2}$; setting

$$
u_{i}=\lambda_{i} \tilde{u}_{i}, \quad i=1,2
$$

we return to the original concentration scales. Relation (16) implies, in particular, that the exclusive-expression steady states $\left(\tilde{u}_{1}, \tilde{u}_{2}\right)=(1,0)$ and $\left(\tilde{u}_{1}, \tilde{u}_{2}\right)=$ $(0,1)$ of the bistable system $(14)-(15)$ map onto $\left(u_{1}, u_{2}\right)=\left(\lambda_{1}, 0\right)$ and $\left(u_{1}, u_{2}\right)=$ $\left(0, \lambda_{2}\right)$ in the original concentration scales $^{1}$.

Figure 3 exemplifies the behaviour of the gene switch model subject to a transition from oscillatory coexpression to the bistable regime by the mechanism described above. The phase plane in panel A includes the coexpression limit cycle of the pre-commitment regime (red colour), as well as a couple of trajectories of the post-commitment bistable regime (black colour), including the separatrix connecting the zero steady state with the coexpression saddle. Importantly, the limit cycle intersects with the separatrix and straddles the post-commitment basins of attraction of the exclusive-expression stable nodes.

The implications of such a configuration are visible from panels B and C, which give the time traces of the free protein concentrations prior to (red colour) and after (black colour) the inducement to commit. The two scenarios coincide in all respects except for the time at which the signal for lineage commitment is given: while in panel $\mathrm{B}$ the signal comes when the first factor peaks, in panel $\mathrm{C}$ the signal arrives as the first factor bottoms out and the second factor is maximal. Upon transitioning into the bistable regime, these two cases are on the opposite sides of the separatrix, leading to opposite attractors being eventually chosen.

The preference for one or the other lineage is decided by the proportion of a complete period $T$ of oscillation that the periodic solution spends in either basin of attraction of the bistable system; we say that the system is being primed for the first $\left(\mathrm{X}_{1}\right)$ or the second $\left(\mathrm{X}_{2}\right)$ factor depending on in which basin it currently resides. In the reference parametric scenario $\left(a_{21}=0.5, a_{12}=0.87, \rho=0.2\right.$, $\left.b_{21}=5, b_{12}=1, \lambda_{1}=\lambda_{2}=3\right)$, the system spends roughly half of the period being primed for either of the two available outcomes (Figure 3, panel D).

\footnotetext{
${ }^{1}$ Transformations of dimensionless quantities (13) and (16) can be derived from a simpler set of transformation rules for the maximal expression rates $p_{i}$ and dissociation constants $K_{i}$ for protein-DNA interaction (see (A1), (A5), and (A6) for relations between dimensional and dimensionless quantities)

$$
\tilde{p}_{i}=\lambda_{i} p_{i}, \quad \tilde{K}_{i}=\lambda_{i} K_{i}, \quad i=1,2 .
$$

Biologically, the required increase in $p_{i}$ means that a committed cell is transcriptionally more active; the increase in $K_{i}$ means that it becomes harder for a transcription factor to interact with its target DNA, e.g. because of increased competition by unspecific binders.
} 

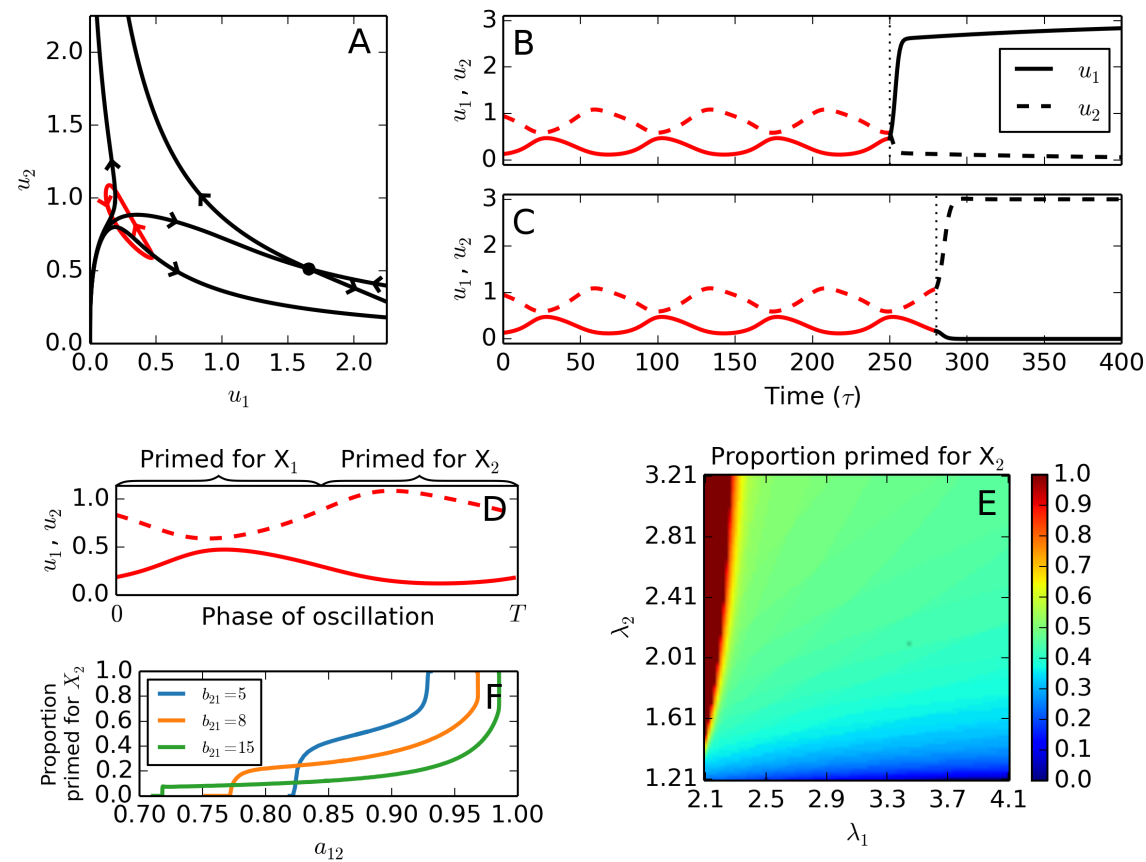

Figure 3: Lineage choice driven by phase. (A) The phase plane includes the limit cycle (red colour) of system (6)-(7) in the oscillatory coexpression regime $\left(a_{21}=\right.$ $0.5, a_{12}=0.87, \rho=0.2, b_{21}=5, b_{12}=1$ ) as well as selected trajectories the bistable system (14)-(15) (black colour) obtained by increasing transcriptional activity threefold $\left(\lambda_{1}=\lambda_{2}=3\right)$. (B-C) Depending on the phase of oscillation at the point of the transition from oscillatory coexpression (red) to bistability (black), either stable steady state can be chosen. (D) Detail of a complete period $T$ of oscillation of the periodic solution to (14)-(15). We indicate the phases spent above the separatrix ("primed for $\mathrm{X}_{2}$ ") and underneath it ("primed for $\left.\mathrm{X}_{1}{ }^{\prime}\right)$. (E-F) The proportion of the period being primed for $\mathrm{X}_{2}$ as function of parameters $\lambda_{1}, \lambda_{2}, a_{12}$ and $b_{21}$ (all parameters are as in panel A unless explicitly stated otherwise). 
By panel E of Figure 3, the proportion primed for $\mathrm{X}_{2}$ increases as function of $\lambda_{2}$ (fold inducement of $\mathrm{X}_{2}$ ) and decreases as function of $\lambda_{1}$ (fold inducement of $\mathrm{X}_{1}$ ). If $\lambda_{1}$ is close (from above) to $1 / a_{21}$, then $\tilde{a}_{21}=\lambda_{1} a_{21}$ is close to one, making the (post-inducement) steady state of exclusive $\mathrm{X}_{1}$ expression marginally stable. The opposite steady state of exclusive $\mathrm{X}_{2}$ expression then attracts the entire (pre-inducement) limit cycle: the solution primes for $X_{2}$ throughout its period of oscillation. Conversely, if $\lambda_{2}$ is close $1 / a_{12}$, the periodic solution primes exclusively for $X_{1}$. Aside from the borderline behaviour, the proportion of time being primed for $\mathrm{X}_{2}$ varies moderately between 0.4 and 0.6 as function of $\lambda_{1}$ and $\lambda_{2}$ (Figure 3, panel E). The proportion of time being primed for the second factor increases with its competition strength $a_{12}$ (Figure 3, panel F); it goes sharply to zero or one close to the Hopf bifurcation points, at which the periodic solution is eliminated (see the next section for details on the bifurcation structure). Away from the bifurcation points, the proportion varies but moderately with $a_{12}$.

Increasing the first factor's binding ability $b_{21}$ typically implies a decrease in the proportion of time that the system is primed for the second factor (Figure 3, panel F). An opposite effect can nevertheless be observed at lower ranges of $a_{12}$, for which the system exhibits low-amplitude oscillations or none, priming exclusively for the first factor; an increase in $b_{21}$ can then amplify the small oscillations, making the second factor available at some phases of the heightened oscillation.

Details on the numerical calculation of the priming proportions are given in Appendix C.

\section{$5 \quad$ Bifurcation structure}

Consider a vertical path in the $\left(a_{21}, a_{12}\right)$-parameter space of the system $(6)-(7)$ (Figure 1, top left), which runs through the point with label 4 in the direction of increasing $a_{12}$. Such a path starts at $a_{12}=0$ in the regime of stable coexpression (Figure 1, top left, yellow region), crosses the oscillatory coexpression regime as $a_{12}$ increases (Figure 1, top left, brown region), and ends in the regime of exclusive expression as $a_{12}$ exceeds one (Figure 1 , top left, white region). The bifurcation diagram in Figure 4 (the left panels, one for either coordinate) details the manner in which these transitions in qualitative behaviour are realised. This bifurcation diagram and the others in this section have been created with the help of the numerical continuation software auto07p (Doedel and Oldeman, 2007).

At $a_{12}=0$, the second factor exerts no competitive effect on the first factor, which is therefore (stably) coexpressed at the maximal possible level $\bar{u}_{1}=1$ while the second factor is coexpressed at a lower level $\bar{u}_{2}=1-a_{21}=0.5$ (Figure 4, left, solid black branch at $a_{12}=0$ ). As the competitive effect $a_{12}$ of the second factor increases from zero to one, the coexpression level $\bar{u}_{1}$ of the first factor decreases, and that of the second factor $\bar{u}_{2}$ increases (Figure 4 , left, the nonconstant steady-state branch). The limit cycle emerges from, and subsequently collapses back into, the coexpression steady state in a pair of supercritical Hopf bifurcations (Figure 4, left, solid red curves). Between the two Hopf bifurcation points $\left(0.82<a_{12}<0.93\right)$, the coexpression steady state loses stability in favour of the limit cycle (Figure 4, left, the dotted part of the nonconstant steady-state branch). At $a_{12}=1$, the coexpression steady state coalesces 

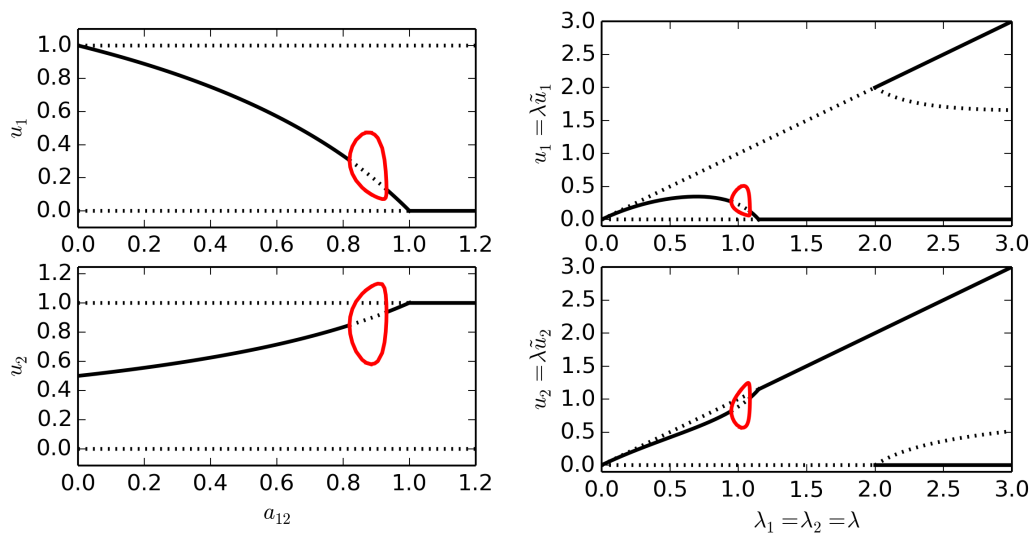

Figure 4: Bifurcation diagrams show the $u_{1^{-}}$(top panels) and $u_{2^{-}}$(bottom panels) coordinates of stable steady states (solid black curves), unstable steady states (dotted black curves), and maxima and minima of stable periodic solutions (solid red curves), as functions of a bifurcation parameter. Implausible steady states with either coordinate negative are omitted from bifurcation diagrams. Left: Bifurcation diagram of the system (6)-(7), in which $a_{12}$ is the bifurcation parameter; $a_{21}=0.5, b_{21}=5, b_{12}=1, \rho=0.2$. Right: Bifurcation diagram of the reparametrised system (14)-(15), in which the fold inducement $\lambda=\lambda_{1}=\lambda_{2}$ is the bifurcation parameter; $a_{21}=0.5, a_{12}=0.87, b_{21}=5$, $b_{12}=1, \rho=0.2$.

with the exclusive-expression steady state of the second factor in a transcritical bifurcation, whereby the two steady states exchange stability (Figure 4, left, $\left.a_{12}=1\right)$. After the transcritical bifurcation, the first coordinate $\bar{u}_{1}$ of the coexpression steady state becomes negative; we discard the implausible coexpression steady-state branch for $a_{12}>1$ from the bifurcation diagram (Figure 4, left, $\left.a_{12}>1\right)$.

The panels on the right-hand side of Figure 4 show a bifurcation diagram for the reparametrised system (14)-(15). The bifurcation parameter $\lambda=\lambda_{1}=\lambda_{2}$ gives the fold increase in competition strengths and binding abilities in (14)-(15) relative to a reference set of parameter values $\left(a_{21}=0.5, a_{12}=0.87, b_{21}=5\right.$, $b_{12}=1, \rho=0.2$ ), see (13). We report the bifurcation diagram in terms of the reference concentration variables $u_{1}=\lambda \tilde{u}_{1}$ and $u_{2}=\lambda \tilde{u}_{2}$, cf. (16), where $\tilde{u}_{1}$ and $\tilde{u}_{2}$ are the dependent variables of the reparametrised system (14)-(15).

The steady-state exclusive-expression concentration of either transcription factor is equal to the bifurcation parameter $\lambda$ (Figure 4, right, the upper steadystate branches in either panel). At $\lambda=1$, we recover the reference system, which possesses a limit cycle, which we previously depicted in Figure 3, panel A (the red orbit). The $\lambda$-diagram (Figure 4, right) is initially structurally similar to the $a_{12}$-diagram (Figure 4 , left), featuring two consecutive supercritical Hopf bifurcations (at $\lambda=0.95$ and $\lambda=1.08$ ) in the coexpression regime and a transcritical bifurcation (at $\lambda=1 / a_{12}=1.15$ ) by which the system transitions into the regime of exclusive expression of the second factor. However, with a further increase in $\lambda$, yet another transcritical bifurcation occurs (at $\lambda=1 / a_{21}=$ 
2 ), this time round at the steady state of exclusive expression of the first factor, whereby it becomes stable and an unstable coexpression steady state re-enters the first quadrant; after the second transcritical bifurcation, the system operates in the bistable regime. At $\lambda=3$, we obtain the bistable system whose phase portrait we previously sketched in Figure 3, panel A (black trajectories).

The stable coexpression branch (Figure 4, solid black branch, $\lambda<1.15$ ), despite the oscillatory intermezzo, is continued after the first transcritical bifurcation by the stable branch of exclusive expression of the (stronger) second factor (Figure 4, solid black branch, $1.15<\lambda<2$ ). The opposite stable branch of exclusive expression of the (weaker) first factor, which enters into play after the second transcritical bifurcation $(\lambda>2)$, can never be reached by following the stable limit sets of the diagram. In order to make both branches available, an instantaneous transition from oscillatory coexpression to the bistable regime is required, as described in Section 4.

The next two bifurcation diagrams, shown in Figures 5 and 6, reveal the typical bifurcation structure exhibited in response to changes in binding abilities $b_{12}, b_{21}$, or the growth rate ratio $\rho$. While the steady-state coordinates of the system (6)-(7) are independent of these three parameters, an increase in the weaker competitor's binding ability or its relative growth rate is conducive to oscillatory coexpression (see Section 3). In Figure 5, we use the standard parameter set used in Figure 1, case 4, and elsewhere, for which the first factor is the weaker competitor, using the first factor's binding ability $b_{21}$ as a bifurcation parameter. A limit cycle emerges from a Hopf bifurcation at $b_{21}=4.37$ and continues to grow in size with further increase in the bifurcation parameter (Figure 5, top left).

For a clear picture of the bifurcation dynamics, we present for selected values of the bifurcation parameter the phase portraits of the system (6)-(7), which include the nullclines shown in blue and green, selected trajectories in black, and, if applicable, the limit cycle in red (Figure 5, top right to bottom). The nullclines consist of trivial branches, which form the boundary of the first quadrant, as well as nontrivial branches within the interior of the first quadrant, the shape of which is parameter-dependent. Except for nongeneric cases, the nontrivial part of either nullcline forms a hyperbola. The $u_{1}$-nullcline, i.e. the set of points on which the concentration of first factor is stationary, is nearly straight and changes very little in response to an increase in its binding ability $b_{21}$ (Figure 5 , blue colour). The $u_{2}$-nullcline, on which the second factor is stationary, undergoes a dramatic transformation as the binding ability of its competitor increases (Figure 5, green colour).

For the degenerate initial case of zero binding ability, $b_{21}=0$, the nontrivial branch of the $u_{2}$-nullcline is a straight line (Figure 5, top right), and the phase portrait is qualitatively identical with that of the competitive species model in the coexistence regime (Murray, 2003). As $b_{21}$ increases, the straight line deforms and assumes a distinctly hyperbolic shape, while the other branch of the hyperbola appears off the $u_{1}$ axis (Figure 5 , centre left). With a further increase in $b_{21}$, the two branches degenerate into their own asymptotes (not shown), after which they reconstitute on the other sides of the asymptotes (Figure 5, centre right). The changes in the $u_{2}$-nullcline are accompanied by the emergence of damped oscillations around the coexpression steady state. These oscillations destabilise after the Hopf bifurcation, and a limit cycle appears, which is initially elliptical (Figure 5, bottom left), but grows in size and bends as $b_{21}$ increases 

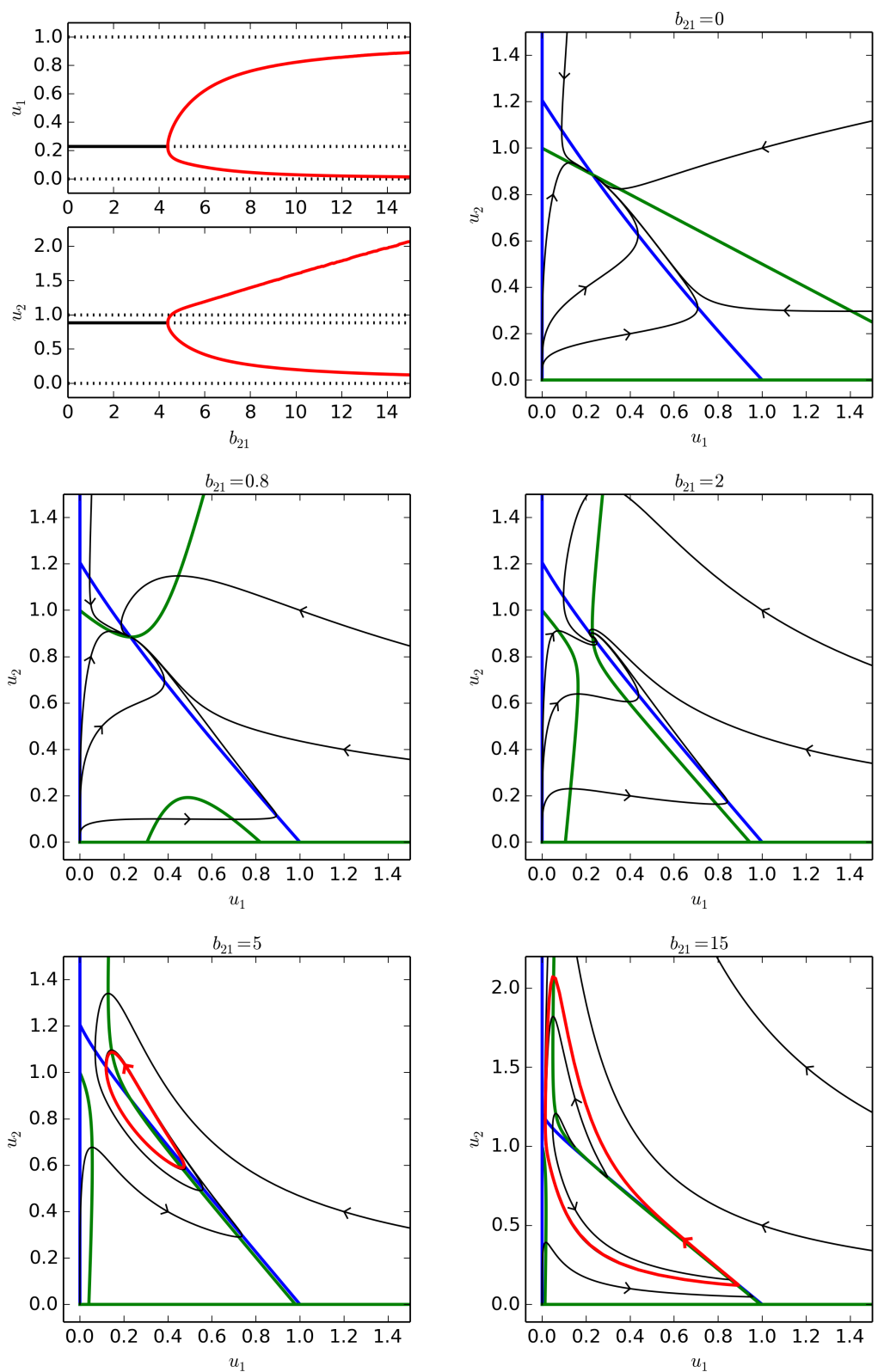

Figure 5: Top Left: Bifurcation diagram of the system (6)-(7), in which $b_{21}$ is the bifurcation parameter; $a_{21}=0.5, a_{12}=0.87, b_{12}=1, \rho=0.2$. Solid curves represent stable limit sets (steady states in black and limit cycles in red); black dotted curves give unstable steady states. Top Right, Centre and Bottom: Phase portraits of (6)-(7) for selected values of $b_{21}$. Other parameters are as in the bifurcation diagram. The portraits include nullclines (in green and blue colours), the limit cycle (red) and selected trajectories (black). 

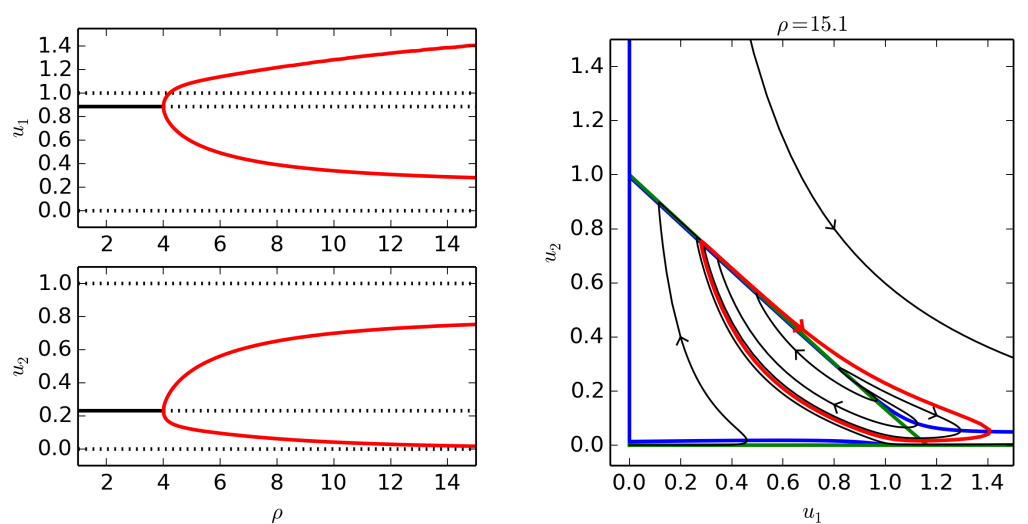

Figure 6: Left: Bifurcation diagram of the system (6)-(7), in which $\rho$ is the bifurcation parameter; $a_{21}=0.87, a_{12}=0.5, b_{21}=1, b_{12}=5$. Solid curves represent stable limit sets (steady states in black and limit cycles in red); black dotted curves give unstable steady states. Right: Phase portrait of $(6)-(7)$ for $\rho=15.1$.

further (Figure 5, bottom right).

The deformation of the $u_{2}$-nullcline reported in the phase portraits in Figure 5 can be related back to our mechanistic understanding of the model (6)-(7). Underneath the $u_{1}$-nullcline, $u_{1}$ grows and captures $u_{2}$ into a complex, reducing or even reversing any growth in $u_{2}$. The reversal of growth in $u_{2}$ occurs where it is a priori weak - near the saturation or extinction points $\left(u_{2}=1\right.$ or $\left.u_{2}=0\right)$ - and where the growth of its capturer is particularly strong (middle values of $u_{1}$ ). These conditions are initially fulfilled in two disconnected components which are delineated by the $u_{2}$-nullcline underneath the $u_{1}$-nullcline (Figure 5 , centre left). After the hyperbolic nullcline branches degenerate and reconstitute on the other sides of their asymptotes, the two components merge into one, while the single component in which $u_{2}$ retains its growth splits into two (Figure 5 , centre right), which subsequently diminish in size as the binding capacity of $u_{1}$ increases further (Figure 5, bottom left). Indeed, if the binding ability of $u_{1}$ is very large, the branches of the $u_{2}$-nullcline become tightly aligned with those of the $u_{1}$-nullcline: except for narrow regions of the phase plane, growth in $u_{1}$ dictates a decrease in $u_{2}$ (Figure 5 , bottom right). Above the $u_{1}$-nullcline, $u_{1}$ decays and the captured $u_{2}$ is released, which brings about a reduction, or even a reversal into growth, of any decline in $u_{2}$. As the binding ability $b_{21}$ of $u_{1}$ increases, the region in which both $u_{1}$ and $u_{2}$ decrease shrinks, until it consists of a narrow strip extending from the coexpression steady state up along the $u_{2}$ axis, where too few $u_{2}$ have been captured to overthrow the tendency to decay. Since growth (decline) in $u_{1}$ implies capture (release) of its competitor $u_{2}$, phases of $u_{1}$-growth overshoot while those of $u_{1}$-decline undershoot the coexpression steady state, thus driving oscillatory behaviour.

Oscillatory behaviour can be reinforced by a fast turnover of $u_{1}$, occurring for $\rho \ll 1$, in which case the capture or release of $u_{2}$ due to growth or decay in $u_{1}$ dominates any slow corrective dynamics of $u_{2}$. The bifurcation structure of the 

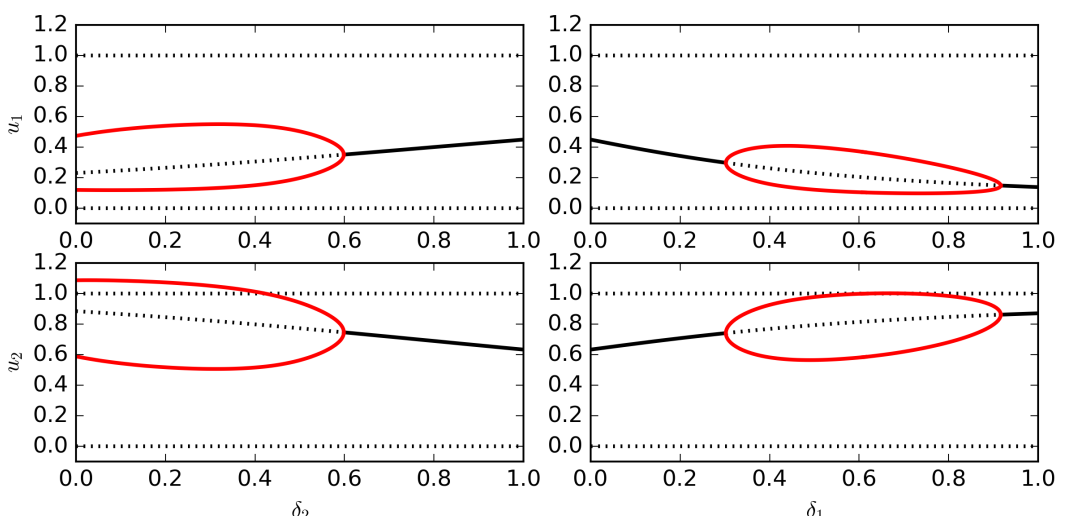

Figure 7: Bifurcation diagrams of (A8)-(A9), in which $\delta_{2}$ (left) or $\delta_{1}(r i g h t)$ is the bifurcation parameter. The other parameters are set to $a_{21}=0.5, a_{12}=$ $0.87, b_{21}=5, b_{12}=1, \rho=0.2, \delta_{1}=0$ (left), $\delta_{2}=1$ (right). Solid curves represent stable limit sets (steady states in black and limit cycles in red); black dotted curves give unstable steady states.

model in response to increasing $1 / \rho$ is similar to the one described above in case of an increasing $b_{21}$ : a limit cycle appears in a supercritical Hopf bifurcation, and continues to grow monotonically in size as the parameter increases. If we exchange the roles of $u_{1}$ and $u_{2}$ by flipping $a_{12} \leftrightarrow a_{21}$ and $b_{12} \leftrightarrow b_{21}$, the same bifurcation structure is obtained in response to increasing the parameter $\rho$ itself (Figure 6).

We have so far studied the phase-plane and bifurcation structure of the system (6)-(7), which was derived in Section 2 from a more general system (A8)(A9) by taking the feedback saturation parameters $\delta_{1}$ and $\delta_{2}$ to zero. Next we use numerical continuation to study the persistence of oscillatory coexpression in (A8)-(A9) as $\delta_{1}$ and $\delta_{2}$ are increased from zero to positive values.

We initially set both $\delta_{1}$ and $\delta_{2}$ to zero whilst keeping the other parameters at values which have previously been shown to support sustained oscillations in the system (6)-(7) (Figure 1, case 4). Increasing $\delta_{2}$ leads to a Hopf bifurcation at $\delta_{2}=0.6$, after which the system no longer supports sustained oscillations (Figure 7, left panels). Fixing $\delta_{2}=1$ and using $\delta_{1}$ as continuation parameter, we observe a re-emergence of the periodic solution after a Hopf bifurcation at $\delta_{1}=0.3$, which collapses back into the coexpression steady state after another Hopf bifurcation at $\delta_{1}=0.92$ (Figure 7 , right panels). Importantly, the results of numerical continuation presented in Figure 7 demonstrate that sustained oscillations are available in the system (A8)-(A9) under medium feedback saturations. 


\section{Discussion}

\subsection{Decay in complex}

We have revisited a mathematical model (Bokes et al., 2009) for a genetic switch that is based on inhibitory protein-protein interactions between two transcription factors, broadly inspired by the pair of hematopoietic regulators PU.1 and GATA-1. Changes have been made to the way protein decay is assumed to act upon protein molecules that are bound to one another: the previous version featured a single mechanism for simultaneous removal of both interacting constituents; here we consider two separate pathways, each targeting one complexed protein species for degradation, freeing the other. As well as encompassing more biological processes, this modification leads to wider possibilities of dynamic behaviour, including the possibility of limit cycle oscillations, which were not reported in (Bokes et al., 2009).

Allowing for degradation of molecules in the complex itself is a prerequisite for obtaining non-trivial behaviour in our model. If, on the contrary, complexification protected its constituents from decay, the transcription factors would not exert any competitive effect on one another, and their concentrations would tend to a simple coexpression stable steady state. We are unaware of any biological evidence directly supporting or contradicting our assumption of degradation in complex. However, we would like to point out that in the well-studied case of the interaction between transcription factors and decoy binding sites a similar assumption has frequently been made (Burger et al., 2010; Lee and Maheshri, 2012; Bokes and Singh, 2015). In case of decoy binding sites a "complex" represents a transcription factor bound to a decoy binding site, but the mathematical representation of such an interaction is virtually identical to ours. Again, it was observed that one has to assume that bound protein molecules are allowed to decay in order to obtain non-trivial behaviour (such as bistability). Without the assumption the model could not explain certain experimental observations (Lee and Maheshri, 2012). We consider it plausible, by analogy, that similar mechanisms could be responsible for maintaining interesting non-equilibrium dynamics in different contexts, in particular in the current lineage-switch model.

\subsection{Model reduction}

Changes to the model (Bokes et al., 2009), and to our insights into what it might demonstrate, prompted us to seek a different choice of nondimensionalisation: the maximal self-sustainable expression levels serve as units of concentration (instead of the interaction's dissociation constant); time is measured in the units of the initial period of growth in the first factor's concentration (instead of the timescale of decay for bound proteins).

Nondimensionalisation helps identify key dimensionless parameter groupings which determine the model's qualitative behaviour. Specifically, there is a parameter $\varepsilon$ which, similarly to its namesake with a slightly different definition in (Bokes et al., 2009), compares the (short) lifetime of individual interactions and the (large) time dynamics of protein concentration accumulation and decay. Following Bokes et al. (2009), we simplified the model by systematically neglecting small $O(\varepsilon)$ terms, obtaining a reduced model (A8)-(A9) of lower order, which can be interpreted as one in which the proportions between free 
and bound molecules adjust instantaneously in response to any changes in total protein concentrations.

There are two other dimensionless parameters, $\delta_{1}$ and $\delta_{2}$, which we consider small here in the analytical work, whereas no analogous assumption was made in (Bokes et al., 2009). Biologically, small $\delta_{i}$ 's imply that the autoregulatory loops of the two transcription factors operate in the low-saturation regime. We concede that this simplifying assumption is motivated primarily by mathematical considerations, rather than biological evidence. Neglecting $O\left(\delta_{i}\right)$ terms facilitates the linearisation analysis of all steady states, including the coexpression one, which was not done in full in (Bokes et al., 2009) and does not seem to be feasible without the simplification. It also helps establish an interesting analogy between lineage switches and a classical ecological model for competitive species, as is discussed below. Nevertheless, we used numerical (continuation) methods to show that the qualitative behaviour of interest, which we identified in the tractable system, persists after $\delta_{i}$ 's are increased to positive values.

\subsection{Lineage switch and competitive species}

Having eliminated $\varepsilon$ and the $\delta_{i}$, our model reduces to (6)-(7), which depend on the five remaining dimensionless quantities: the competition coefficients $a_{12}$ and $a_{21}$, the binding capacities $b_{12}$ and $b_{21}$, and the ratio $\rho$ of initial growth rate constants.

The value of $a_{12}$ measures the competitive effect of the second on the first factor: the steady-state exclusive expression of the second factor is stable with respect to perturbations adding small amounts of the first factor only if $a_{12}$ exceeds one. For high values of $b_{12}$, the competitive effect is due to the capture by the second factor of most molecules of the first factor into a complex. Low values of $b_{12}$ imply that only a small fraction of the first factor's molecular concentration becomes bound by the second factor, and any competition results from an elevated propensity for degradation in bound state.

If $b_{12}=b_{21}=0$, then, rather than forming an interaction, random collisions of molecular pairs lead to immediate annihilation of either factor. Mathematically, our model (6)-(7) then formally reduces to a system that has traditionally been used in mathematical ecology to describe the population dynamics of competitive species (Murray, 2003). It is well known that the competitive-species model operates a bistable regime if both competition coefficients exceed one, a coexpression regime if both are less than one, and a regime of the stronger competitor's exclusivity in the remaining cases of highly asymmetric competition. We have demonstrated that the same coarse-grained classification remains valid even if $b_{12}$ or $b_{21}$ are nonzero.

However, a number of additional observations that hold for the competitive species model - the limited impact of $\rho$ on the qualitative behaviour and the impossibility of either damped or sustained oscillations - no longer apply if $b_{12}$ or $b_{21}$ are allowed to be positive. Linearising the model around the coexpression steady state in the coexpression regime shows that the steady state can become unstable in certain parametric regimes, implying that a limit cycle must exist which attracts solutions repelled by the unstable state. These oscillatory regimes are characterised by parametric asymmetry: in order that limit-cycle coexpression occurs, it is necessary that: 
- competition coefficients are unequal (and both less than one);

- the weaker competitor has a greater binding capacity and/or grows faster, but is more available for degradation in bound form.

These results reinforce previous observations that asymmetry in parameter values can supply additional functionality to lineage switches (Alagha and Zaikin, 2013). They allow us to speculate that alternating lineage promiscuity in multipotent progenitor cells can be realised by deterministic oscillations sustained by a lineage switch in the regime of weak cross-inhibition.

Deterministic oscillations can alternatively be sustained by intransitive competition between three or more transcription factors (May and Leonard, 1975; Rabajante and Babierra, 2015; Rabajante and Gavina, 2015).

\subsection{Limit-cycle coexpression and lineage promiscuity}

The bistable regime offers two stable steady states in which either transcription factor is exclusively expressed. Each steady state is associated with commitment to a distinct cell fate. The model also provides two coexpression regimes: one is realised by a globally stable steady state; the other is characterised by the existence of a stable limit cycle. The former can account for simultaneous, and the latter for alternating lineage promiscuity.

Lineage selection can be represented by an (instantaneous) parametric change from a coexpression into the bistable regime. If coexpression is realised by a globally stable steady state, then the choice of attractor in the bistable regime depends solely on which basin of attraction the coexpression state appears in after a transition into bistable regime. The choice is therefore predetermined by the nature of the transition: one lineage is inevitable and the other is impossible. Nevertheless, both outcomes can be made available if one considers stochastic effects. Stationary behaviour of a stochastic model will not be concentrated in the single point of stable coexpression steady state, but will instead be distributed in an ellipsoid around it, as is dictated by the fluctuation-dissipation theorem (Paulsson, 2004). If the steady state is positioned close to the separatrix delineating the basins of attraction, then - even in small-noise conditions - the ellipsoid will transcend the basins, enabling either attractor to be selected (Andrecut et al., 2011).

As an alternative to the above mechanism, we propose to use the limit cycle which is available in our model to account for alternating lineage promiscuity. Both basins of attraction in the bistable regime contain a section of the limit cycle. Therefore, depending on the phase of the oscillation at the point of transition into bistability, either attractor can eventually be selected. The proportion attaining each outcome can be assigned by appropriate choice of parameter values (see Section 4).

We considered a specific type of parameter transition to bistability which involved a proportional increase in the transcription factors' competition strengths, binding abilities, and maximal self-sustainable leves. Biologically, a positive value of the proportionality factor $\lambda$ can be achieved by a coordinated increase in the maximal transcription rates and dissociation constants for protein-promoter interactions. Increasing transcription rates has traditionally been used as a mechanism for resolving a lineage switch (Laslo et al., 2006; Antebi et al., 2013; Roeder and Glauche, 2006). Increases in the dissociation constants could result 
from a supposed rise in unspecific molecular competition at the promoter (Matsuda et al., 2014). Bifurcation analysis has previously been used to characterise the progress of lineage selection (Huang et al., 2007; Li et al., 2015). We used the induction factor $\lambda$, among other parameters, in the continuation of the limit cycle of the model (Section 5).

It has previously been argued (e.g. by Huang et al. (2007) and Foster et al. (2009)) that at least three concurrent stable limit sets (a tristable regime) are required in a regulatory motif governing lineage selection, with one stable state corresponding to multilineage priming and at least two stable states representing the alternatives in cell-fate selection. Tristability could be obtained in our model by making the bifurcation parameter $\lambda$ dependent on the resosution of an upstream primary genetic switch or a "preswitch" (Laslo et al., 2006; Schittler et al., 2010). The preswitch would be required to possess two stable steady states, one characterised by a low value and the other by a high value of $\lambda$. These two values would induce coexpression and bistability regimes, respectively, in the secondary switch, which would be described by our current model. Taken together, the combined motif consisting of a primary and the secondary switch would possess three limit sets: a single limit set would exhibit a low value of $\lambda$ and coexpress the two factors of the secondary switch (through a stable steady state or a limit cycle); additionally, there would be two stable steady states with a high value of $\lambda$, exclusively expressing either transcription factor of the secondary switch. Alternatively, the model could be made tristable without a primary switch by including cooperativity in the transcription factors' positive feedback. However, it is not immediately clear (and would be worth investigating in the future) how adding cooperativity affects the oscillatory behaviour of the model.

In conclusion, our model provides a mechanism for selection of cell fate using oscillations in a purely deterministic model for two antagonistic transcription factors. More widely, it suggests that protein-protein interactions can sustain interesting dynamical behaviour in genetic regulatory networks. Additionally, it illustrates that perturbation methods can be used to examine parallels between detailed law-of-mass-action models of chemical kinetics and simpler (often phenomenological) systems traditionally used in population dynamics and mathematical ecology.

\section{Appendix A: Nondimensionalisation and simpli- fication of the model}

In addition to the trivial zero steady state, the system given by (5) and (3) has a steady state in which the first factor is expressed at a non-zero level, $x_{1}=p_{1} / \alpha_{1}-K_{1}$, while its antagonist, and the complex, are absent $\left(x_{2}=y=\right.$ $0)$, as well as the symmetric reflection of that steady state, which is given by $x_{2}=p_{2} / \alpha_{2}-K_{2}, x_{1}=y=0$. We assume that $p_{i} / \alpha_{i}-K_{i}>0, i=1,2$, so that both are physically admissible. Other steady states may also exist, namely coexpression ones for which $x_{1}>0, x_{2}>0$, and $y>0$ hold simultaneously. These are hard to investigate by analytic methods; however, progress can be made after a series of rational approximations, which are performed below. 
We nondimensionalise (5) and (3) according to

$$
t=\frac{\tau}{\frac{p_{1}}{K_{1}}-\alpha_{1}}, \quad x_{i}=\left(\frac{p_{i}}{\alpha_{i}}-K_{i}\right) u_{i}, \quad y=\frac{\left(\frac{p_{1}}{\alpha_{1}}-K_{1}\right)\left(\frac{p_{2}}{\alpha_{2}}-K_{2}\right)}{K_{d}} v
$$

in which $K_{\mathrm{d}}=k_{\mathrm{off}} / k_{\mathrm{on}}$, obtaining

$$
\begin{aligned}
\frac{\mathrm{d}\left(u_{1}+b_{12} v\right)}{\mathrm{d} \tau} & =\frac{u_{1}\left(1-u_{1}\right)}{1+\delta_{1} u_{1}}-a_{12} v, \\
\frac{\mathrm{d}\left(u_{2}+b_{21} v\right)}{\mathrm{d} \tau} & =\rho\left(\frac{u_{2}\left(1-u_{2}\right)}{1+\delta_{2} u_{2}}-a_{21} v\right), \\
\varepsilon \frac{\mathrm{d} v}{\mathrm{~d} \tau} & =u_{1} u_{2}-v-\varepsilon\left(\frac{a_{12}}{b_{12}}+\rho \frac{a_{21}}{b_{21}}\right) v,
\end{aligned}
$$

where

$$
\begin{aligned}
& a_{12}=\frac{\beta_{1} K_{1}\left(\frac{p_{2}}{\alpha_{2}}-K_{2}\right)}{\alpha_{1} K_{d}\left(\frac{p_{1}}{\alpha_{1}}-K_{1}\right)}, \quad a_{21}=\frac{\beta_{2} K_{2}\left(\frac{p_{1}}{\alpha_{1}}-K_{1}\right)}{\alpha_{2} K_{d}\left(\frac{p_{2}}{\alpha_{2}}-K_{2}\right)}, \\
& b_{12}=\frac{\frac{p_{2}}{\alpha_{2}}-K_{2}}{K_{d}}, \quad b_{21}=\frac{\frac{p_{1}}{\alpha_{1}}-K_{1}}{K_{d}}, \quad \rho=\frac{\frac{p_{2}}{K_{2}}-\alpha_{2}}{\frac{p_{1}}{K_{1}}-\alpha_{1}}, \\
& \delta_{1}=\frac{p_{1}}{\alpha_{1} K_{1}}-1, \quad \delta_{2}=\frac{p_{2}}{\alpha_{2} K_{2}}-1, \quad \varepsilon=\frac{\frac{p_{1}}{K_{1}}-\alpha_{1}}{k_{\text {off }}}
\end{aligned}
$$

are dimensionless parameters. The parameter $\varepsilon$ is the ratio of the growth rate constant of the first factor - the first eigenvalue of the linearisation around the trivial steady state - to the rate constant for complex dissociation. Typically, the timescale of protein accumulation would be much slower than the lifetime of individual protein-protein interactions, implying that $\varepsilon$ is a small parameter.

Taking $\varepsilon \rightarrow 0$ in (A4), we obtain $v=u_{1} u_{2}$ which, if inserted into (A2)-(A3), leads to a two-dimensional system for $u_{1}$ and $u_{2}$,

$$
\begin{aligned}
& \frac{\mathrm{d}\left(u_{1}+b_{12} u_{1} u_{2}\right)}{\mathrm{d} \tau}=u_{1}\left(\frac{1-u_{1}}{1+\delta_{1} u_{1}}-a_{12} u_{2}\right), \\
& \frac{\mathrm{d}\left(u_{2}+b_{21} u_{1} u_{2}\right)}{\mathrm{d} \tau}=\rho u_{2}\left(\frac{1-u_{2}}{1+\delta_{2} u_{2}}-a_{21} u_{1}\right) .
\end{aligned}
$$

The three-dimensional problem (A2)-(A4) is singularly perturbed (Kevorkian and Cole, 1981) in $\varepsilon$. Should initial conditions be imposed on it, say at $\tau=0$, a separate analysis is required to obtain the correct leading-order behaviour at the $\tau=O(\varepsilon)$ transient timescale (Bokes et al., 2009). The single most important feature of the behaviour will be a fast relaxation of the three-component solution onto the two-dimensional "slow manifold" (Jones, 1995) given by $v=u_{1} u_{2}$.

If $p_{1} / \alpha_{1}-K_{1}=0$, the original dimensional system given by (5) and (3) undergoes a transcritical bifurcation on the $x_{1}$-axis, whereby the exclusiveexpression steady state coalesces with the trivial zero steady state. An analogous bifurcation occurs on the $x_{2}$-axis if $p_{2} / \alpha_{2}-K_{2}=0$. The closeness of the system to these bifurcation points is characterised by the dimensionless parameters $\delta_{i}(\mathrm{~A} 7)$, which compare the steady-state values $p_{i} / \alpha_{i}-K_{i}$ of exclusive expression to the effective dissociation constant $K_{i}$ for non-cooperative autoregulation. 
The chosen nondimensionalisation (A1) allows us to focus on the behaviour of the system in the vicinity of these bifurcations. Neglecting the $O\left(\delta_{1}\right)$ and $O\left(\delta_{2}\right)$ terms in $(\mathrm{A} 8)-(\mathrm{A} 9)$, we obtain the final system $(6)-(7)$, which describes the limiting near-bifurcation behaviour. We emphasise that taking $\delta_{1}, \delta_{2} \rightarrow 0$ in (A8)-(A9) (or, equivalently, earlier in (A2)-(A4)) is different from taking $p_{i} / \alpha_{i}-K_{i}=0$ in (5): while the former yields the limiting near-bifurcation behaviour, the latter recovers the behaviour exactly at the bifurcation point.

The effective dissociation constant $K_{i}$ gives the concentration of protein that is required by the positive feedback loop to achieve the half-maximal rate of protein production. A small value of $\delta_{i}$ means that only a small fraction of the maximal production rate is realised at steady state, and that the protein expression is sustained by an undersaturated feedback loop. While we do not claim that such feedback loops are necessarily prevalent, the smallness of the $\delta_{i}$ greatly facilitates the tractability of the model, motivating us to make this assumption.

\section{Appendix B: Linearisation in neighbourhoods of steady states}

In the analysis that follows, $\overline{\boldsymbol{u}}=\left(\bar{u}_{1}, \bar{u}_{2}\right)$ can represent any of the four available steady states: the zero steady state $(0,0)^{\top}$; two steady states of exclusive expression $(1,0)^{\top}$ and $(0,1)^{\top}$; the coexpression steady state (11). Consider a time-dependent solution which is close to the steady state:

$$
\boldsymbol{u}(\tau)=\overline{\boldsymbol{u}}+\epsilon \tilde{\boldsymbol{u}}(\tau), \quad \epsilon \ll 1 .
$$

Inserting (B1) into (6)-(7) and neglecting higher-order terms we obtain a linear system

$$
\boldsymbol{B} \frac{\mathrm{d} \tilde{\boldsymbol{u}}}{\mathrm{d} \tau}=\boldsymbol{A} \tilde{\boldsymbol{u}}
$$

where

$$
\boldsymbol{A}=\left(\begin{array}{cc}
1-2 \bar{u}_{1}-a_{12} \bar{u}_{2} & -a_{12} \bar{u}_{1} \\
-\rho a_{21} \bar{u}_{2} & \rho\left(1-2 \bar{u}_{2}-a_{21} \bar{u}_{1}\right)
\end{array}\right)
$$

and

$$
\boldsymbol{B}=\left(\begin{array}{cc}
1+b_{12} \bar{u}_{2} & b_{12} \bar{u}_{1} \\
b_{21} \bar{u}_{2} & 1+b_{21} \bar{u}_{1}
\end{array}\right) .
$$

System (B2) assumes a diagonal structure for the zero steady state and a triangular structure for either exclusive-expression steady state. Such structures make the task of finding eigenvalues, to determine stability, quite easy: the results are summarised in the Main Text.

The analysis is only slightly more complicated for the coexpression steady state. Since it satisfies $1-\bar{u}_{1}-a_{12} \bar{u}_{2}=1-\bar{u}_{2}-a_{21} \bar{u}_{1}=0$, the diagonal terms of matrix (B3) simplify to

$$
\boldsymbol{A}=-\left(\begin{array}{cc}
\bar{u}_{1} & a_{12} \bar{u}_{1} \\
\rho a_{21} \bar{u}_{2} & \rho \bar{u}_{2}
\end{array}\right) .
$$

The determinant of the linearisation matrix in (B2) satisfies

$$
\operatorname{det}\left(\boldsymbol{B}^{-1} \boldsymbol{A}\right)=\frac{\operatorname{det}(\boldsymbol{A})}{\operatorname{det}(\boldsymbol{B})}=\frac{\rho \bar{u}_{1} \bar{u}_{2}\left(1-a_{12} a_{21}\right)}{1+b_{12} \bar{u}_{2}+b_{21} \bar{u}_{1}} .
$$


If $a_{12}>1$ and $a_{21}>1$, then $\operatorname{det}\left(\boldsymbol{B}^{-1} \boldsymbol{A}\right)<0$, implying that (B2) has two real eigenvalues of opposite signs, and the coexpression steady state is a saddle. If $a_{12}<1$ and $a_{21}<1$, we have $\operatorname{det}\left(\boldsymbol{B}^{-1} \boldsymbol{A}\right)>0$, implying that the coexpression state cannot be a saddle: it is a node or a spiral; its stability is determined by the sign of

$$
\begin{aligned}
\operatorname{tr}\left(\boldsymbol{B}^{-1} \boldsymbol{A}\right) & =\frac{\rho \bar{u}_{2}\left(a_{21} b_{12} \bar{u}_{1}-1-b_{12} \bar{u}_{2}\right)+\bar{u}_{1}\left(a_{12} b_{21} \bar{u}_{2}-1-b_{21} \bar{u}_{1}\right)}{1+b_{12} \bar{u}_{2}+b_{21} \bar{u}_{1}} \\
& =\frac{\rho \bar{u}_{2}\left(b_{12}\left(1-2 \bar{u}_{2}\right)-1\right)+\bar{u}_{1}\left(b_{21}\left(1-2 \bar{u}_{1}\right)-1\right)}{1+b_{12} \bar{u}_{2}+b_{21} \bar{u}_{1}},
\end{aligned}
$$

which yields the condition for instability (12).

\section{Appendix C: Calculating priming proportions}

In order to determine the proportion of time spent by the periodic solution in either basin of attraction, we need to calculate the oscillating solution to (6)-(7) and its period; we also need to calculate the separatrix of the post-commitment system (14)-(15) and implement an automated test which decides for a given point in the phase plane which side of the separatrix it resides. Below we describe how we carried out each of these tasks.

Rather than integrating an initial-value problem and checking for return to the initial condition, we determined the periodic solution to (6)-(7) on one complete period of oscillation using a boundary-value approach with the continuation software auto07p (Doedel and Oldeman, 2007). The numerical solution returned by auto07p is defined on a nonuniform time discretisation of the period, which is denser where the solution moves faster. We used linear interpolation (using Python's interp1d from the scipy.interpolate package) to obtain the values of the solution on a uniform time discretisation. For each of these values, we tested, using a procedure described below, which side of the separatrix of the post-commitment system (14)-(15) it falls into: the proportion of these values found in the basin of attraction of the second factor was returned as the numerical approximation of the proportion of the period spent in the basin.

After the signal for commitment is given, the transcription factors are governed by system (14)-(15), which is the same system as (6)-(7) but with changed parameters (13) and concentration scales (16). For notational simplicity, we show how to find a separatrix for system (6)-(7) operating in the bistable regime; we then comment on the transformations that are required to use this procedure to obtain the separatrix for (14)-(15).

The separatrix consists of two (up to a time shift) solutions to (6)-(7) which approach the saddle point as time increases. We focus exclusively on cases when the separatrix forms a graph of a function $u_{2}=S\left(u_{1}\right)$ (such as in Figure 1, bistable regime). Dividing (9) by (8), we obtain for the function $u_{2}=S\left(u_{1}\right)$ a first-order differential equation

$$
\frac{\mathrm{d} u_{2}}{\mathrm{~d} u_{1}}=\frac{-b_{21} u_{2} f_{1}\left(u_{1}, u_{2}\right)+\left(1+b_{12} u_{2}\right) f_{2}\left(u_{1}, u_{2}\right)}{\left(1+b_{21} u_{1}\right) f_{1}\left(u_{1}, u_{2}\right)-b_{12} u_{1} f_{2}\left(u_{1}, u_{2}\right)},
$$

where $f_{1}\left(u_{1}, u_{2}\right)$ and $f_{2}\left(u_{1}, u_{2}\right)$ are given by (10). The separatrix passes through 
the saddle point with coordinates

$$
\bar{u}_{1}=\frac{1-a_{12}}{1-a_{12} a_{21}}, \quad \bar{u}_{2}=\frac{1-a_{21}}{1-a_{12} a_{21}},
$$

for which $f_{1}\left(\bar{u}_{1}, \bar{u}_{2}\right)=f_{2}\left(\bar{u}_{1}, \bar{u}_{2}\right)=0$ holds, so that the right-hand side of $(\mathrm{C} 1)$ is not defined there. Avoiding the saddle point, we solve (C1) numerically on the interval $0<u_{1}<\bar{u}_{1}-\kappa_{1}$, where $\kappa_{1} \ll 1$, subject to a terminal condition

$$
u_{2}=\bar{u}_{2}-\kappa_{1} \frac{v_{2}}{v_{1}} \text { at } u_{1}=\bar{u}_{1}-\kappa_{1},
$$

where $v_{1}$ and $v_{2}$ are the coordinates of the eigenvector corresponding to the negative eigenvalue of the linearisation around the saddle point of the system (6)-(7). The terminal-value problem $(\mathrm{C} 1)$ and $(\mathrm{C} 2)$ amounts to an initial-value problem in $-u_{1}$. We also solve (C1) numerically on the interval $\bar{u}_{1}+\kappa_{2}<u_{1}<1$, where $\kappa_{2} \ll 1$, subject to an initial condition

$$
u_{2}=\bar{u}_{2}+\kappa_{2} \frac{v_{2}}{v_{1}} \quad \text { at } u_{1}=\bar{u}_{1}+\kappa_{2} .
$$

Concatenating the two solutions, we obtain a numerical approximation of the separatrix $u_{2}=S\left(u_{1}\right)$ defined on a fine discretisation of the interval $0<u_{1}<1$. We use linear interpolation (again Python's interp1d) to obtain the $S\left(u_{1}\right)$ for any value from within the unit interval; values of $S\left(u_{1}\right)$ outside of the unit interval are not needed. We classify a given point $\left(u_{1}, u_{2}\right)$ in the phase plane such that $0<u_{1}<1$ and $u_{2}>0$ as belonging to the basin of attraction of the stable steady state $(0,1)$ if $u_{2}>S\left(u_{1}\right)$ holds; otherwise it belongs to the basin of attraction of $(1,0)$.

Applying the above procedure on the post-commitment system (14)-(15) leaves us with a (numerical representation of) the separatrix $\tilde{u}_{2}=\tilde{S}\left(\tilde{u}_{1}\right)$ in the post-commitment concentration scales; using (16), we obtain

$$
u_{2}=S\left(u_{1}\right)=\lambda_{2} \tilde{S}\left(\lambda_{1}^{-1} u_{1}\right)
$$

for the separatrix in the pre-commitment concentration scales (in which the periodic solution is recorded).

\section{Acknowledgement}

Pavol Bokes acknowledges the support of the Slovak Research and Development Agency (grant no. APVV-14-0378), the VEGA Grant Agency (grant no. $1 / 0319 / 15)$, and the European Commission through Marie Curie Early Stage Researcher Training (contract no. MEST-CT-2005-020723). We thank the reviewers for helpful suggestions and relevant references.

\section{References}

Akashi, K., Traver, D., Miyamoto, T., and Weissman, I. (2000). A clonogenic common myeloid progenitor that gives rise to all myeloid lineages. Nature, 404:193-197. 
Alagha, A. and Zaikin, A. (2013). Asymmetry in erythroid-myeloid differentiation switch and the role of timing in a binary cell-fate decision. Front. Immunol., 4:426.

Alon, U. (2007). An Introduction to Systems Biology: Design Principles of Biological Circuits. Chapman \& Hall/CRC.

Alsaedi, A., Zaikin, A., Ahmad, B., Alsaadi, F., and El-Shahed, M. (2014). Fractional calculus model of gata-switching for regulating the differentiation of a hematopoietic stem cell. Adv. Differ. Equ-ny., 2014(1):201.

Andrecut, M., Halley, J., Winkler, D., and Huang, S. (2011). A general model for binary cell fate decision gene circuits with degeneracy: Indeterminacy and switch behavior in the absence of cooperativity. PloS one, 6:e19358.

Antebi, Y. E., Reich-Zeliger, S., Hart, Y., Mayo, A., Eizenberg, I., Rimer, J., Putheti, P., Pe'er, D., and Friedman, N. (2013). Mapping differentiation under mixed culture conditions reveals a tunable continuum of $\mathrm{T}$ cell fates. Plos Biology, 11:e1001616.

Bintu, L., Buchler, N., Garcia, H., Gerland, U., Hwa, T., Kondev, J., and Phillips, R. (2005). Transcriptional regulation by the numbers: models. Curr. Opin. Genet. Dev., 15:116-124.

Bokes, P., King, J., and Loose, M. (2009). A bistable genetic switch which does not require high co-operativity at the promoter: a two-timescale model for the PU.1-GATA-1 interaction. Math. Med. Biol., 26:117-132.

Bokes, P., King, J., Wood, A., and Loose, M. (2013). Transcriptional bursting diversifies the behaviour of a toggle switch: hybrid simulation of stochastic gene expression. B. Math. Biol., 75:351-371.

Bokes, P. and Singh, A. (2015). Protein copy number distributions for a selfregulating gene in the presence of decoy binding sites. PloS one, 10:e120555.

Buggenthin, F., Buettner, F., Hoppe, P. S., Endele, M., Kroiss, M., Strasser, M., Schwarzfischer, M., Loeffler, D., Kokkaliaris, K. D., Hilsenbeck, O., et al. (2017). Prospective identification of hematopoietic lineage choice by deep learning. Nat. Methods, 14(4):403-406.

Burger, A., Walczak, A. M., and Wolynes, P. G. (2010). Abduction and asylum in the lives of transcription factors. P. Natl. Acad. Sci. USA, 107:4016-4021.

Cantor, A. and Orkin, S. (2001). Hematopoietic development: a balancing act. Curr. Opin. Genet. Dev., 11:513-519.

Cao, Y., Lopatkin, A., and You, L. (2016). Elements of biological oscillations in time and space. Nat. Struct. Mol. Biol., 23:1030-1034.

Chen, H., Ray-Gallet, D., Zhang, P., Hetherington, C., Gonzalez, D., Zhang, D., Moreau-Gachelin, F., and Tenen, D. (1995). PU.1 (Spi-1) autoregulates its expression in myeloid cells. Oncogene, 11:1549-1560. 
Chickarmane, V., Enver, T., and Peterson, C. (2009). Computational modeling of the hematopoietic erythroid-myeloid switch reveals insights into cooperativity, priming, and irreversibility. PLoS Comput. Biol., 5:e1000268.

Doedel, E. J. and Oldeman, B. E. (2007). AUTO-07P: continuation and bifurcation software for ordinary differential equations. Concordia University, Montreal.

Duff, C., Smith-Miles, K., Lopes, L., and Tian, T. (2012). Mathematical modelling of stem cell differentiation: the PU.1-GATA-1 interaction. J. Math. Biol., 64:449-468.

Erban, R., Kevrekidis, I., Adalsteinsson, D., and Elston, T. (2006). Gene regulatory networks: a coarse-grained, equation-free approach to multiscale computation. J. Chem. Phys., 124:084106.

Foster, D. V., Foster, J. G., Huang, S., and Kauffman, S. A. (2009). A model of sequential branching in hierarchical cell fate determination. J. Theor. Biol., 260:589-597.

Graf, T. (2002). Differentiation plasticity of hematopoietic cells. Blood, 99:30893101.

Hirsch, M. W. (1982). Systems of differential equations which are competitive or cooperative: I. limit sets. SIAM J. Math. Anal., 13:167-179.

Hoppe, P. S., Schwarzfischer, M., Loeffler, D., Kokkaliaris, K. D., Hilsenbeck, O., Moritz, N., Endele, M., Filipczyk, A., Gambardella, A., Ahmed, N., et al. (2016). Early myeloid lineage choice is not initiated by random PU. 1 to GATA1 protein ratios. Nature, 535(7611):299-302.

Hu, M., Krause, D., Greaves, M., Sharkis, S., Dexter, M., Heyworth, C., and Enver, T. (1997). Multilineage gene expression precedes commitment in the hemopoietic system. Genes Dev., 11:774-785.

Huang, S., Guo, Y., May, G., and Enver, T. (2007). Bifurcation dynamics in lineage-commitment in bipotent progenitor cells. Dev. Biol., 305:695-713.

Jones, C. K. R. T. (1995). Geometric singular perturbation theory. In Dynamical systems, pages 44-118. Springer.

Keener, J. and Sneyd, J. (2008). Mathematical Physiology: Cellular Physiology. Springer.

Kevorkian, J. and Cole, J. (1981). Perturbation Methods in Applied Mathematics. Springer.

Krumsiek, J., Marr, C., Schroeder, T., and Theis, F. J. (2011). Hierarchical differentiation of myeloid progenitors is encoded in the transcription factor network. PloS one, 6:e22649.

Kulessa, H., Frampton, J., and Graf, T. (1995). GATA-1 reprograms avian myelomonocytic cell lines into eosinophils, thromboblasts, and erythroblasts. Genes Dev., 9:1250-1262. 
Laslo, P., Spooner, C., Warmflash, A., Lancki, D., Lee, H., Sciammas, R., Gantner, B., Dinner, A., and Singh, H. (2006). Multilineage transcriptional priming and determination of alternate hematopoietic cell fates. Cell, 126:755-766.

Lee, T.-H. and Maheshri, N. (2012). A regulatory role for repeated decoy transcription factor binding sites in target gene expression. Mol. Syst. Biol., 8:576.

Li, S., Liu, Y., Liu, Z., and Wang, R. (2015). Bifurcation dynamics and determination of alternate cell fates in bipotent progenitor cells. Cogn. Neurodynamics, 9:221-229.

Matsuda, H., Putzel, G. G., Backman, V., and Szleifer, I. (2014). Macromolecular crowding as a regulator of gene transcription. Biophys. J., 106:1801-1810.

May, R. M. and Leonard, W. J. (1975). Nonlinear aspects of competition between three species. SIAM J. Appl. Math., 29:243-253.

McDevitt, M., Fujiwara, Y., Shivdasani, R., and Orkin, S. (1997). An upstream, DNase I hypersensitive region of the hematopoietic-expressed transcription factor GATA-1 gene confers developmental specificity in transgenic mice. $P$. Natl. Acad. Sci. USA, 94:7976-7981.

Mitra, M. K., Taylor, P. R., Hutchison, C. J., McLeish, T., and Chakrabarti, B. (2014). Delayed self-regulation and time-dependent chemical drive leads to novel states in epigenetic landscapes. J. Roy. Soc. Interface, 11:20140706.

Murray, J. (2003). Mathematical Biology: I. Introduction. Springer.

Nerlov, C. and Graf, T. (1998). PU.1 induces myeloid lineage commitment in multipotent hematopoietic progenitors. Genes Dev., 12:2403-2412.

Nimmo, R. A., May, G. E., and Enver, T. (2015). Primed and ready: understanding lineage commitment through single cell analysis. Trends Cell Biol., $25: 459-467$.

Paulsson, J. (2004). Summing up the noise in gene networks. Nature, 427:415418.

Rabajante, J. F. and Babierra, A. L. (2015). Branching and oscillations in the epigenetic landscape of cell-fate determination. Prog. Biophys. Mol. Biol., 117:240-249.

Rabajante, J. F. and Gavina, M. K. A. (2015). Producing oscillatory decisions. Neurosci. Commun., 2:e859.

Roeder, I. and Glauche, I. (2006). Towards an understanding of lineage specification in hematopoietic stem cells: a mathematical model for the interaction of transcription factors GATA-1 and PU.1. J. Theor. Biol., 241:852-865.

Ruggieri, A., Dazert, E., Metz, P., Hofmann, S., Bergeest, J.-P., Mazur, J., Bankhead, P., Hiet, M.-S., Kallis, S., Alvisi, G., et al. (2012). Dynamic oscillation of translation and stress granule formation mark the cellular response to virus infection. Cell Host Microbe, 12:71-85. 
Schittler, D., Hasenauer, J., Allgöwer, F., and Waldherr, S. (2010). Cell differentiation modeled via a coupled two-switch regulatory network. Chaos, 20:045121.

Shea, M. and Ackers, G. (1985). The OR control system of bacteriophage lambda: a physical-chemical model for gene regulation. J. Mol. Biol., 181:211230 .

Shivdasani, R. and Orkin, S. (1996). The transcriptional control of hematopoiesis. Blood, 87:4025-4039.

Strasser, M., Theis, F. J., and Marr, C. (2012). Stability and multiattractor dynamics of a toggle switch based on a two-stage model of stochastic gene expression. Biophys. J., 102:19-29.

Swiers, G., Patient, R., and Loose, M. (2006). Genetic regulatory networks programming hematopoietic stem cells and erythroid lineage specification. Dev. Biol., 294:525-540.

Teles, J., Pina, C., Edén, P., Ohlsson, M., Enver, T., and Peterson, C. (2013). Transcriptional regulation of lineage commitment-a stochastic model of cell fate decisions. PLoS Comput. Biol., 9:e1003197.

Tian, T. and Smith-Miles, K. (2014). Mathematical modeling of GATAswitching for regulating the differentiation of hematopoietic stem cell. Bmc Syst. Biol., 8:S8.

Tyson, J., Chen, K., and Novak, B. (2003). Sniffers, buzzers, toggles and blinkers: dynamics of regulatory and signaling pathways in the cell. Curr. Opin. Cell Biol., 15(2):221-231.

Velten, L., Haas, S. F., Raffel, S., Blaszkiewicz, S., Islam, S., Hennig, B. P., Hirche, C., Lutz, C., Buss, E. C., Nowak, D., et al. (2017). Human haematopoietic stem cell lineage commitment is a continuous process. Nat. Cell Biol.

Waters, R. S., Perry, J. S., Han, S., Bielekova, B., and Gedeon, T. (2017). The effects of interleukin-2 on immune response regulation. Math. Med. Biol., page dqw021.

Zhang, P., Behre, G., Pan, J., Iwama, A., Wara-aswapati, N., Radomska, H., Auron, P., Tenen, D., and Sun, Z. (1999). Negative cross-talk between hematopoietic regulators: GATA proteins repress PU.1. P. Natl. Acad. Sci. USA, 96:8705-8710.

Zhang, P., Zhang, X., Iwama, A., Yu, C., Smith, K., Mueller, B., Narravula, S., Torbett, B., Orkin, S., and Tenen, D. (2000). PU.1 inhibits GATA-1 function and erythroid differentiation by blocking GATA-1 DNA binding. Blood, 96:2641-2648. 